\title{
Preventing growth faltering among Australian Indigenous children: implications for policy and practice
}

\author{
Elizabeth L McDonald, Ross S Bailie, Alice R Rumbold, Peter S Morris and Barbara A Paterson
}

O ur review was commissioned as a result of an international trend to encourage public policy and management to be better informed by the results of relevant and reliable research. ${ }^{1}$ The need for this comes from research showing that some health and social interventions believed to be beneficial were in fact harmful; some interventions, although expensive, were largely ineffective; and some effective inventions have been adopted only slowly or ignored. ${ }^{2}$

Frequently, the incorporation of interventions into policy is influenced more by different perceptions of the seriousness and cause of problems (held by politicians, the general public, and influential individuals or organisations) rather than the evidence available. ${ }^{3}$ This is very much the case in developing policies and programs to address the health and other problems confronting Indigenous Australians. The subject of our review is the problem of growth faltering, and subsequent failure to thrive, among Australian Indigenous children. The causes of this problem are multiple and complex. There are no simple or quick solutions to achieve sustainable reductions in prevalence.

The normal growth curve of children is represented as gradual, incremental increases in weight from birth based on regular recordings of bodyweight. ${ }^{4}$ Growth faltering is a reduction in the expected rate of growth along an infant's previously defined growth curve. In contexts of poverty, growth faltering typically occurs at about 6 months of age with children's transition to foods that are often inadequate in quantity and quality. In addition, the children experience greater exposure to the environment and the increased likelihood of illness. ${ }^{5}$ Preventing growth faltering is important, as early childhood growth and development set a base for subsequent learning, behaviour and health over the life cycle. ${ }^{6}$

In the Northern Territory, rates of underweight among Indigenous children are slowly declining. However, Indigenous children under 5 years in remote NT communities still experience high levels of underweight (14.5\%), stunting (11.3\%) and wasting $(9.0 \%)^{7}$ In a healthy population profile, the expected prevalence of underweight is $\leqslant 2.3 \%{ }^{7}$ A complex mix of factors, including poor nutrition, acute and chronic infections and parasitic diseases, combines to hinder the healthy growth of Indigenous children in remote communities. ${ }^{8}$ Addressing the problems that underlie these conditions and the level of disadvantage experienced by Australian Indigenous people presents a significant challenge to policymakers. We undertook a systematic literature review to determine what preventive models or programs are most likely to improve patterns of growth faltering in children aged under 5 years in remote Indigenous communities.

\section{Methods}

Our methods were designed to take account of the likely causes of growth faltering among Australian Indigenous children living in remote communities. We searched nine electronic databases and the websites of key stakeholder, government and non-government agencies. All types of study design were eligible.

The first stage focused on identifying relevant systematic reviews, the second on identifying relevant primary studies, and the third on

\begin{abstract}
Objective: To determine what preventive models or programs are most likely to improve patterns of growth faltering in children aged under 5 years in remote Australian Indigenous communities.

Methods: Nine electronic databases and the websites of key stakeholder, government and non-government agencies were searched. Two reviewers independently assessed articles for inclusion and for study quality. All types of study design were eligible.
\end{abstract}

Results: 140 studies assessing a diverse range of interventions were identified. Of these, 51 articles referring to 44 individual programs and 7 review articles met the review criteria. The evidence for the effectiveness of many interventions to prevent growth faltering is not strong, and any observed effects are modest. Community-based nutrition education/counselling and multifaceted interventions involving carers, community health workers and community representatives, designed to meet program best-practice requirements and address the underlying causes of growth faltering, may be effective in preventing growth faltering. Other interventions, such as food distribution programs, growth monitoring, micronutrient supplementation and deworming should only be considered in the context of broader primary health care programs and/or when there is an identified local need.

Conclusion: For remote Indigenous communities, development and implementation of programs should involve a consideration of the evidence for potential impact, strength of community support and local feasibility. Given the lack of strong evidence supporting programs, any new or existing programs require ongoing evaluation and refinement.

MJA 2008; 188: S84-S86

identifying relevant reports in the grey literature. Two reviewers independently assessed articles for inclusion and for study quality. We adapted existing data extraction forms and dictionaries to assess eligibility for inclusion and study quality. ${ }^{9,10}$ Primary outcomes measured included:

- Proportion of children with wasting (ie, with weight more than 2 SD below the median weight for height or less than $80 \%$ of standard weight for height) at 2 years of age;

- Mean difference in weight gain at 2 years of age.

Full details of the methods used are available in our final report. ${ }^{11}$

\section{Results}

We found that growth faltering may be prevented by communitybased nutrition education/counselling and interventions consisting of more than one strategy that involve carers, community health workers and community representatives; that are designed to meet program best-practice requirements; and that address the underlying causes of growth faltering (Box 1). 
We found that current evidence supporting interventions to prevent growth faltering is limited by a lack of good quality data. Few programs assessed have focused on Australian Indigenous children. Key issues emerging from the review process that weakened the evidence included failing to identify and address the direct and indirect causes of growth faltering among the research population, as well as not reporting the social, economic, environmental and other contextual factors that would impact on the success or otherwise of interventions.

\section{Discussion}

A key implication of our findings is reflected in a recent report on policies of Australian governments in the broad area of child health and wellbeing. ${ }^{12}$ The report identifies better coordination of existing policies as the priority issue for child health. The report also highlights the need to identify any gaps in existing policy. These actions are necessary because child health policies are being developed in several sectors and implemented across several portfolios (for example health, welfare and education), as well as through several levels of government.

This results in a fragmented approach to program development and service delivery, which in turn makes it difficult for those working at community level to take a strategic approach to addressing Indigenous child health problems. This is particularly evident when these problems arise from a mix of complex issues such as poor living conditions, overcrowding, low socioeconomic status, family and community dysfunction, and poor access to early intervention and social support services.

The implications for policy and practice from the research evidence arising from this review are:

- Interventions consisting of more than one strategy, including community-based nutrition education/counselling, that (a) involve carers, community health workers and community representatives; (b) are designed to meet program best-practice requirements (specifically including the requirement that interventions be developed through participatory processes involving community stakeholders); (c) address the underlying causes of growth faltering, and focus on nutrition behaviour change; and (e) are well integrated into primary health care systems, may prevent growth faltering. These interventions require ongoing evaluation and refinement.

- In general, community feeding programs should only be implemented in situations in which food insecurity is a major problem and in which feeding programs are supported by the local community. Such programs should only be seen as a relatively short-term solution.

- Growth monitoring can be included in programs that aim to prevent failure to thrive. However, there is a need to ensure that this intervention is integrated into a broader primary health care program that includes assessment of other major risks to child health. These programs need to ensure early follow-up and effective action when growth faltering is detected. In particular, there is a need to treat common infections and specific nutrient deficiencies and to ensure that an appropriate quantity of suitable food is available to the child on an ongoing basis.

- Vitamin and mineral supplementation (iron, zinc, vitamin A, multiple micronutrients) should only be considered in populations in which a deficiency is known to be a common underlying cause of growth faltering.

- Deworming as a means of preventing growth faltering should be used in populations known to suffer from high rates of infestation, and should be used in association with other interventions that will prevent reinfestation (eg, improved sanitation and hand-washing).

These potential interventions rely on some understanding of the factors that underlie growth faltering in a particular community. The relative significance of individual factors is likely to vary between communities. Box 2 provides a framework to guide policy for improving the growth and development of young Indigenous children and preventing disease. Policy and programs have tended to "medicalise" the problem of growth faltering, the causes of which are now recognised as being largely social and economic in origin. $^{14}$

Several important implications for policy and practice were drawn from the general literature, including the following:

- There is an ongoing need to strengthen resources and capacity at the community level to plan and implement preventive programs using culturally appropriate and participatory methods, with a focus on the family.

\section{Review findings}

The nature of the interventions to prevent growth faltering that have been the subject of research is diverse. Interventions, used singly or in combination, have included food supplements (for children and pregnant or lactating women), growth monitoring, education/ counselling (for carers of children, community workers or health workers), deworming, vitamin and mineral supplements, multiple micronutrient supplements, and studies that simultaneously address more than one underlying factor.

Approaches used to implement interventions have included centrebased programs and home visiting. The quality of research evidence for interventions is variable and generally poor. Many reports focus on specific interventions rather than program models, and the reporting of information on intervention design and implementation is generally inadequate. The major findings of our review were as follows:

1. While the evidence is not strong and the effects are modest, the interventions for which there is some evidence of benefit in general populations include:

- Community-based nutrition/counselling interventions that focus on nutrition behaviour change and interventions that are well integrated into primary health care systems.

2. Interventions for which there is some evidence of benefit in specific populations include:

- Vitamin A supplementation in populations with moderate-tosevere vitamin A deficiency, and deworming treatment in populations with high infestation rates.

3. Interventions for which the research evidence clearly supports neither implementation of new programs nor withdrawal of existing programs* include:

- Supplementary/complementary feeding programs for children or pregnant/lactating women and multiple micronutrient supplementations.

4. Interventions where the research showed no clear evidence of benefit include:

- Growth-monitoring programs, population iron supplementation for children, and iron and zinc supplementation in general. There was clear evidence that zinc supplementation was of no benefit in preventing growth faltering. However, supplementation was associated with increases in height.

* Interventions for which there is no evidence of benefit or harm (in some studies, there were methodological concerns). 


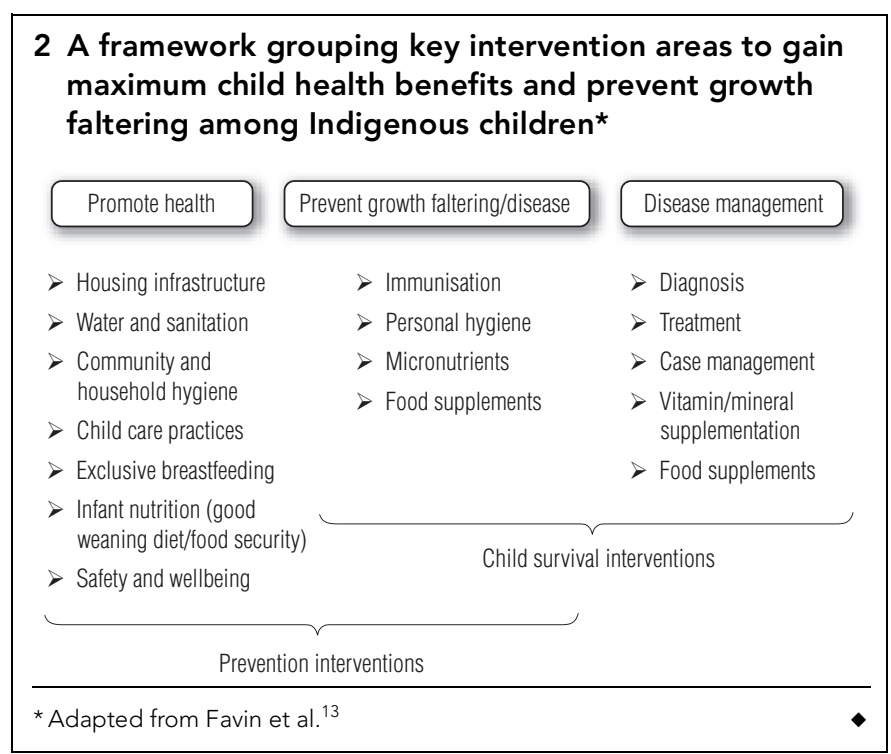

- Interventions should be selected on the basis of evidence of potential impact and on feasibility in the local context.

- The importance of many factors that are more or less outside the influence of the health sector highlights the need for good coordination and cooperation between agencies to develop and implement comprehensive programs.

- There is a need to enhance the acceptability of interventions to caregivers, families and the wider community in order to effectively engage with families on a continuing basis.

Hence, while the underlying causes of growth faltering in a remote community should inform the choice of intervention(s), the approach taken to implementing interventions needs to be acceptable to community members.

The complex nature of growth faltering (and the importance of the problem) is reflected in the diversity of interventions identified and assessed in our review. It is clear that the serious consequences of growth faltering require immediate measures to limit its impact, as well as longer term measures to address the underlying causes of the problem. It is critical that interventions to prevent growth faltering among Australian Indigenous children living in remote communities address the complex mix of social, cultural, economic and environmental factors that underlies the problem.

There is a clear need for high-quality evidence to support specific preventive interventions, in addition to better evidence on approaches to implementing interventions. This is especially relevant to remote Indigenous communities, where the primary cause of disadvantage is largely related to social exclusion and poverty. International experience indicates that coordinating interventions between the health sector and other sectors will do more to improve children's health in contexts of poverty than a series of single interventions, or than one sector trying to address the problem alone. ${ }^{15}$ Strengthening resources and capacity at the community level to plan and implement preventive programs that are culturally appropriate, participatory, and focus on the family, appears to be the key to success and sustainability of interventions.

\section{Competing interests}

None identified.

\section{Author details}

Elizabeth L McDonald, PhD, Research Fellow, Services, Systems and Society Division ${ }^{1}$

Ross S Bailie, MD, MPhil(MCH), FAFPHM, Professor of Public Health and Program Leader, Comprehensive Primary Health Care Team ${ }^{1}$

Alice R Rumbold, BSc(Hons), MPH, PhD, Postdoctoral Research Fellow, Services, Systems and Society Division ${ }^{1}$

Peter S Morris, MB BS, FRACP, PhD, Head, Ear Health and Education Program, ${ }^{1}$ Senior Lecturer of Clinical Epidemiology, ${ }^{2}$ and Senior Paediatrician ${ }^{3}$

Barbara A Paterson, FFPHM, MPH, MRCGP, Program Director ${ }^{4}$

1 Menzies School of Health Research, Charles Darwin University, Darwin, NT.

2 NT Clinical School, Flinders University, Darwin, NT.

3 Royal Darwin Hospital, Darwin, NT.

4 Maternal and Child Health Division, Northern Territory Department of

Health and Community Services, Darwin, NT.

Correspondence: liz.mcdonald@menzies.edu.au

\section{References}

1 Black N, Mays $N$, editors. Synthesizing evidence for management and policy-making. J Health Serv Res Policy 2005; 10 Suppl 1.

2 Sheldon T. Making evidence synthesis more useful for management and policy-making. J Health Serv Res Policy 2005; 10 Suppl 1: 1-5.

3 Oliver TR. The politics of public health policy. Annu Rev Public Health 2006; 27: 195-233.

4 World Health Organization Global database on child growth and malnutrition. Geneva: WHO, 1997. http://www.who.int/nutgrowthdb/en (accessed Jan 2008).

5 Caulfield LE, Richard SA, Rivera JA, et al. Stunting, wasting, and micronutrient deficiency disorders. In: Jamison DT, Breman JG, Measham AR, et al, editors. Disease control priorities in developing countries. 2nd ed. New York: World Bank/Oxford University Press, 2006: 551-567.

6 Grantham-Mcgregor SM, Walker SP, Chang S. Nutritional deficiencies and later behavioural development. Proc Nutr Soc 2000; 59: 47-54.

$7 \mathrm{Li}$ SQ, Guthridge SL, Tursan d'Espaignet E, Paterson BA. From infancy to young adulthood: health status in the Northern Territory, 2006. Darwin: Department of Health and Community Services, 2007. http:// www.nt.gov.au/health//health_gains/epidemiology/from_infancy_to_ young_adulthood_health_status_in_the_northern_territory_2006.pdf (accessed Jan 2008).

8 Brewster D. Failure to thrive. In: Couzos S, Murray R, editors. Aboriginal primary health care: an evidence-based approach. 2nd ed. Melbourne: Oxford University Press, 2003: 162-185.

9 health-evidence.ca [website]. Validity tool: review articles. http://healthevidence.ca/pdf/ValidityTool.pdf (accessed Aug 2007).

10 Cochrane Effective Practice and Organisation of Care Review Group. The data collection checklist. Aberdeen: Health Services Research Unit, Department of Public Health, University of Aberdeen, 2002.

11 McDonald E, Bailie R, Morris $P$, et al. Interventions to prevent growth faltering in remote Indigenous communities. Canberra: Australian Primary Health Care Research Institute and Menzies School of Health Research, 2006. http://www.anu.edu.au/aphcri/Domain/ATSIPHC/Final_ 25_Bailie.pdf (accessed Jan 2008).

12 National Health and Medical Research Council. Recent and current policies of Australian governments in the broad area of child health and wellbeing. Canberra: NHMRC, 2006.

13 Favin $M$, Yacoob M, Bendahmane DB, editors. Behavior first: a minimum package of environmental health behaviors to improve child health. Report No.10. Washington: Environmental Health Project, 1999.

14 Brewster DR. Critical appraisal of the management of severe malnutrition: 4. Implications for Aboriginal child health in northern Australia. J Paediatr Child Health 2006; 42: 594-595.

15 Hill Z, Kirkwood B, Edmond K. Family and community practices that promote child survival, growth and development: a review of the evidence. Geneva: World Health Organization, 2004. 